\title{
Retrobulbar Optic Neuritis Associated with Tacrolimus Decrease after Allogeneic hematopoietic Stem Cell Transplantation for Acute Myeloblastic Leukemia Patient
}

\author{
S Schulmann', C Pochon ${ }^{1}$, J Colne ${ }^{2}$, F Trechot ${ }^{2}$, K Angioi ${ }^{2}$, S Pittion-Vouyovitch ${ }^{3}$, C Bonmati' ${ }^{1}$ G Roth-

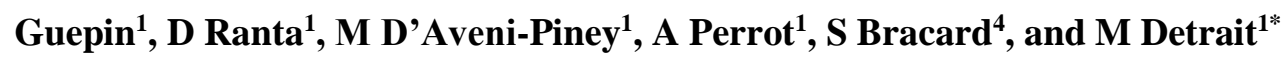

${ }^{1}$ Hematology Department, CHRU de Nancy, Hôpitaux de Brabois, Vandoeuvre-lès-Nancy, France

${ }^{2}$ Ophtalmology Department, CHRU de Nancy, Hôpitaux de Brabois, Vandoeuvre-lès-Nancy, France

${ }^{3}$ Neurology Department, CHRU de Nancy, Hôpital Central, Nancy, France

${ }^{4}$ Neuro-Radiology Department, CHRU de Nancy, Hôpital Central, Nancy, France

*Corresponding author: Marie Detrait, Hematology Department, CHRU de Nancy, Hôpitaux de Brabois, rue du Morvan, 54500 Vandoeuvre-lès-Nancy, France, Email: m.detrait@chru-nancy.fr

\begin{abstract}
Neurological complications are common after allogeneic hematopoietic stem cell transplantation (HSCT) but it is often difficult to define the cause among infections, Graft- versus-Host Disease (GvHD), adverse effects from medications or nervous system disease (for instance autoimmune disease). We describe a rare case of ocular GvHD associated with the reduction of tacrolimus in a female patient, three months after allogeneic HSCT for acute myeloblastic leukemia in second remission. The multidisciplinary investigations led to the diagnosis of retrobulbar optic neuritis associated with the expression of auto (allo)immune markers. Quickly after rising dosage of tacrolimus to therapeutic level, the ocular symptoms disappeared without relapse thereafter.
\end{abstract}

Keywords: Allogeneic hematopoietic stem cell transplantation; Immune markers; Retrobulbar optic neuritis

Received Date: March 17, 2018; Accepted Date: April 8, 2018; Published Date: April 15, 2018

\section{Introduction}

Chronic Graft- versus-Host Disease (cGvHD) is a major complication altering patients' quality of life after allogeneic HSCT. According to the National Institute of Health, the diagnosis of cGvHD requires 3 items: 1) distinction from acute GvHD, 2) presence of at least 1 diagnostic clinical sign of cGvHD or presence of at least 1 distinctive manifestation confirmed by pertinent biopsy or other relevant tests (for instance new onset of dry, gritty or painless eyes, cicatricial conjunctivitis, keratoconjunctivitis sicca and confluent aeras of punctuate keratopathy) and 3) exclusion of other possible diagnosis [1]. Neurological manifestations of cGvHD are diverses: immune-mediated neuropathies, myasthenia gravis and myositis in the peripheral nervous system and various cerebrovascular complications, demyelination and immune-mediated encephalitis in the

Citation: Detrait M. Retrobulbar Optic Neuritis Associated with Tacrolimus Decrease after Allogeneic hematopoietic Stem Cell Transplantation for Acute Myeloblastic Leukemia Patient. J Clin Cases Rep 2018: 1(1) 30-35. DOI: https://doi.org/10.46619/joccr.2018.1$\underline{1007}$ 
www.tridhascholars.org | April-2018

central nervous system [2]. This article describes sudden visual loss in the left eye after tacrolimus decrease, in a female patient, three months after undergoing allogeneic HSCT for acute Myeloblastic leukemia.

\section{Case Presentation}

A forty-seven-year-old woman was diagnosed with acute myeloid leukemia in June 2014. She was treated for hypothyroidism and hypertension with a 30 pack-year smoking history. She had never experienced neurological or ocular injuries. She had been treated in 2013 for an adenocarcinoma in the left breast by complete mastectomy followed by hormonal therapy with tamoxifene. Reconstructive surgery was scheduled but the preoperative checkup revealed $33 \%$ of circulating myeloid blasts. The initial disease assessment concluded a normal karyotype and a mutation of NPM1 without FLT3 internal tandem duplication. The patient underwent induction chemotherapy with Daunorubicine $\left(60 \mathrm{mg} / \mathrm{m}^{2}\right.$ day $1-3$ and $35 \mathrm{mg} / \mathrm{m}^{2}$ day $\left.8-9\right)$ and Cytarabine $\left(500 \mathrm{mg} / \mathrm{m}^{2}\right.$ days $1-3$ and $1000 \mathrm{mg} / \mathrm{m}^{2}$ BID days 8-10) on June 12, 2014 followed by three consolidation therapies with Cytarabine (3000 mg/m² BID days 1,3,5) leading to a first complete remission in December 2014.

On July 2015, a blood sample revealed pancytopenia with circulating blasts. The relapse was confirmed in the bone marrow. There were still no abnormalities on the karyotype and NPM1 mutation was present again. The treatment consisted on one cure of Cytarabine (1000 mg/m² BID days 1-5), Mitoxantrone (12 mg/m² days 1-3), and Gemtuzumab Ozogamicin (9 mg/m² day 4) leading to a second complete remission and followed by one consolidation treatment with Cytarabine (200 mg/m² days $1-5)$ and Gemtuzumab (6 mg/m² on day 1). The indication of allogeneic HSCT was set and a compatible HLA donor was sought.

A 9/10 HLA-matched unrelated donor was identified on the registers of bone marrow voluntary donors. There was a mismatch on HLA-A locus and two mismatches on DPB1 locus (11/14 HLA). The transplantation was performed on November $26^{\text {th }}$ 2015. Pre-transplantation conditioning included Fludarabine $\left(30 \mathrm{mg} / \mathrm{m}^{2}\right)$ for 6 days and Busulfan $(3,2 \mathrm{mg} / \mathrm{kg})$ for $2 \mathrm{days}$. A reduced intensity conditioning was selected because the HCT-CI-age score was high at 8 (active smoking, left ventricular ejection fraction was $50 \%$ with septal hypokinesis, DLCO $60 \%$, history of breast cancer and age) but the young age of patient led us to maintain the project of allogeneic HSCT. The GvHD prophylaxis was provided by antithymocyte globulins (ATG) 5 $\mathrm{mg} / \mathrm{kg}$ distributed on D-3, D-2, D-1, Methotrexate on D+1, D+3, D+5, because of ABO minor incompatibility and tacrolimus from D-1. Febrile neutropenia was controlled by ceftazidim and vancomycin with a good outcome. Complete response was achieved, confirmed with a $100 \%$ donor chimerism on bone marrow after recovery of aplasia. The patient hasn't shown any sign of acute GvHD and did not receive prophylactic donor lymphocyte infusion.

Three months after transplantation, the dose of tacrolimus was decreasing progressively when the patient suddenly experienced painless visual loss in the left eye. She underwent an ophthalmologic examination who revealed visual acuity of $1 / 10$, no Tyndall effect or hyalitis, neither papillary edema nor ischemia. Neurological exam was normal except for a Marcus Gunn left pupil sign. Macular and pupillary optical coherence tomography showed no abnormalities. Cerebral magnetic resonance imaging (MRI) revealed T2 hyper intensity of the left optic nerve with contrast reaction of its intra-canalicular portion, which was consistent with the diagnosis of left inflammatory Retrobulbar optic neuritis (Figure 1). Doppler ultrasonography of the supra aortic vessels was normal. Immune investigations found positive antinuclear antibodies (ANA 128) and rheumatoid factor (RF 46.3 UI/mL). There was no argument in favor of infection or toxicity. Subsequently, the patient presented with oral lichen plan us suspect of cGvHD, which justified local treatment and increasing the dosage of tacrolimus to obtain a residual therapeutic level again, followed by complete visual remission within 3 days. Concerning residual plasma levels of tacrolimus, 
before the episode, the plasma level was at a therapeutic level (T0 at $13.5 \mu \mathrm{g} / \mathrm{L}$ with tacrolimus at $1 \mathrm{mg} /$ day).When the loss of vision occurred, the decrease of tacrolimus had just started 15 days ago and we did not achieve residual plasma levels. After a complete recovery of the ocular symptoms, $\mathrm{T} 0$ was at $6.5 \mu \mathrm{g} / \mathrm{L}$ with a dose of $1.5 \mathrm{mg} / \mathrm{day}$ of tacrolimus thus a therapeutic rate.

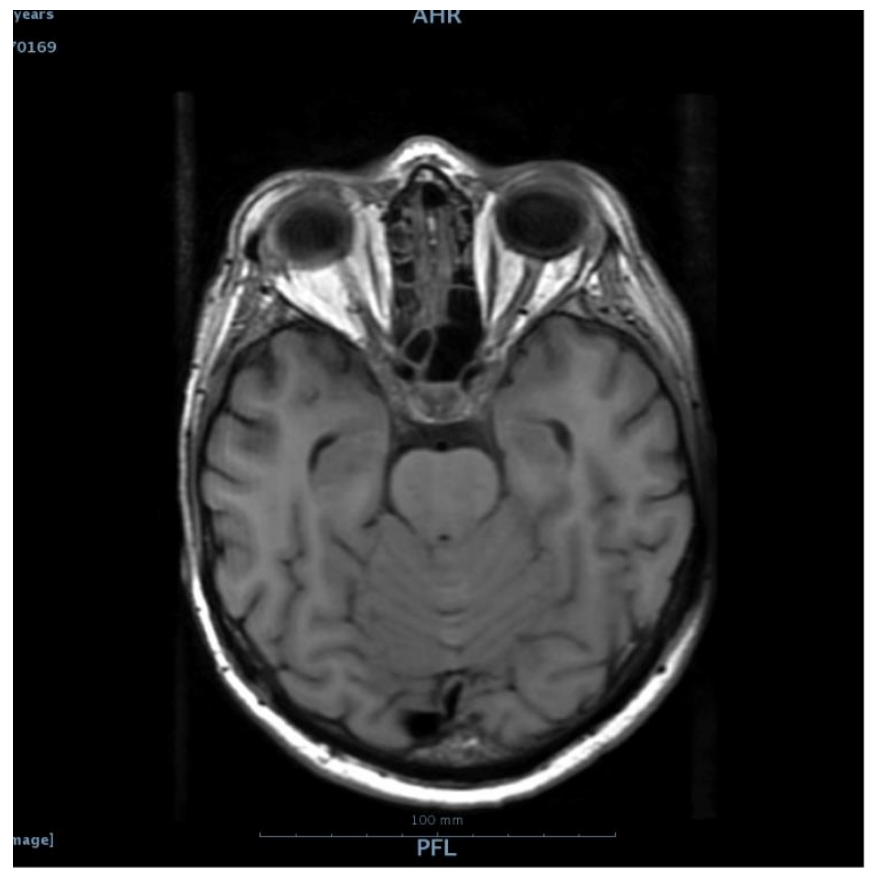

Figure 1: T1 axial without injection: hyper signal of the left optic nerve, intraorbital portion

Thereafter, she hasn't been experiencing any ocular relapse; a slow decrease of tacrolimus was initiated in September 2016 at $1 \mathrm{mg}$ per day in 2 takes. One year later, there was no recurrence of any neurological or ocular symptoms but the low-grade of cGvHD with dry syndrome of oral cavity was persisting with a mild hypereosinophilia on the latest blood test. In this context, the dosage of tacrolimus hadn't been reduced since the end of 2016. In the last news, chimerism on blood was still $100 \%$ donor and NPM1 mutation on blood was undetected in November 2017.

\section{Discussion}

Considering no other cause for the ocular condition of the patient, the presence of cGvHD in the mouth and the improvement with immunomodulatory therapy, we consider cGvHD to have been the cause of retro bulbar optic neuritis in our patient. Several international clinical trials suggest that chronic GvHD is a T-cell mediated disease because of a lower incidence in the setting of T-cell depleted graft. And more recently, it has been shown that B-cells are also involved in this pathophysiology confirmed by the presence of circulating autoantibodies (antinuclear, anti-DNA) in some patients' serum. The standard firstline treatment remains prednisolone initiated at a dose of $1-2 \mathrm{mg} / \mathrm{kg} / \mathrm{day}[3,4]$.

Acute disseminated encephalomyelitis (ADEM) has been described in patients following allogenic HSCT along with GvHD, suggesting that donor immune T-cells infiltrate the central nervous system (CNS) leading to an auto(allo)immune entity called CNS-GvHD by Harvey et al. [5] (Figure 2 and Figure 3). 


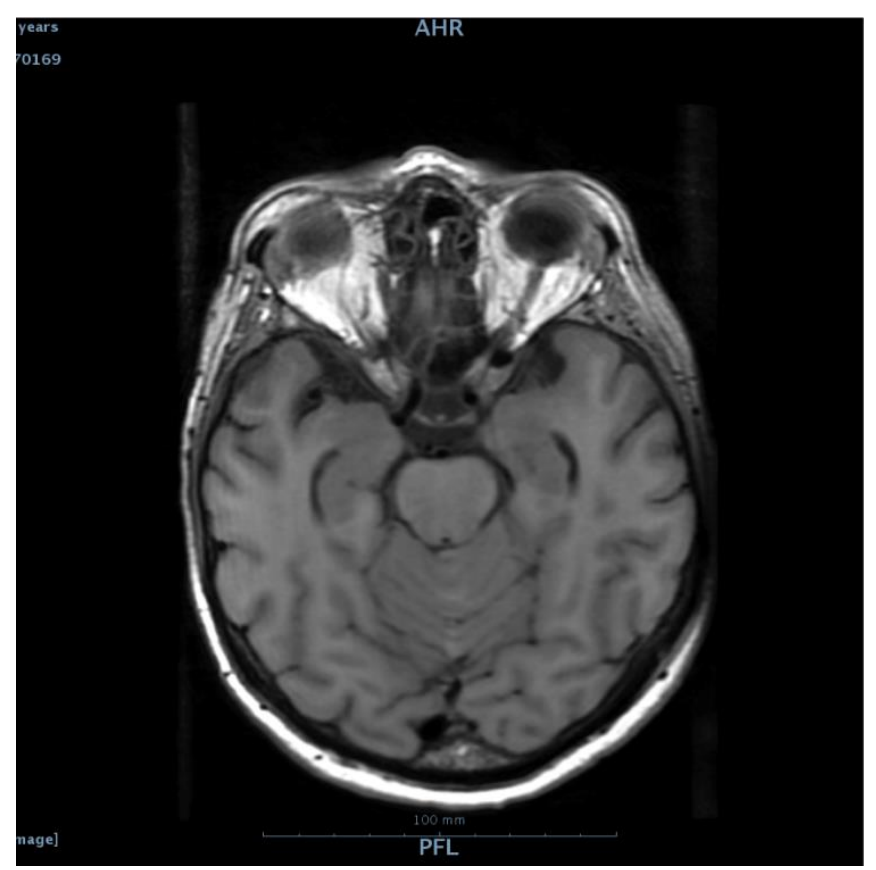

Figure 2: T1 axial without injection: hyper signal of the left optic nerve in the intracranial section in the cavernous sinus The clinical features of ADEM concern the timing and location of the symptoms, including encephalopathy, behavior changes or consciousness alteration and no alternatives causes.

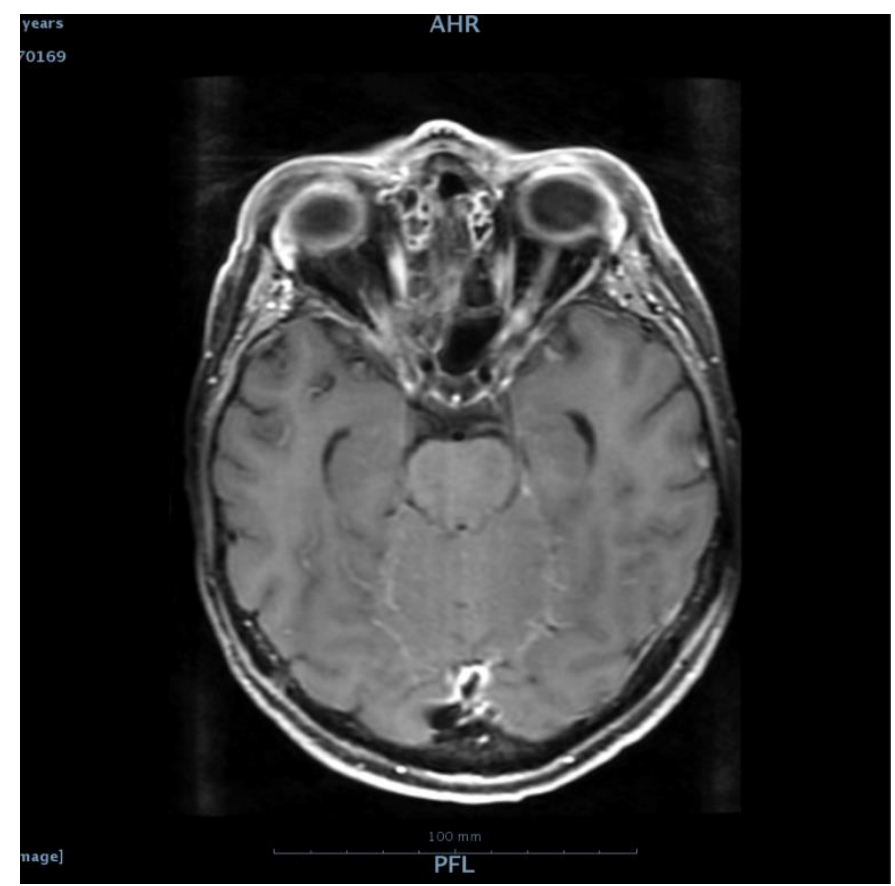

Figure 3: T1axial with Gadolinium injection: enhancement of the left optic nerve in the intraorbital part.

The MRI shows large, multifocal, hyper intense lesions in T2 and Flair sequences. In Pittock's article, this phenomenon is attributed to inflammation, which induces T-cells activation, responsible for hypersensivity reaction in neurons [6]. 
Optic neuritis (ON) is mainly idiopathic but it can be related to demyelinating injuries in multiple sclerosis (MS) or neuromyelitis optica (NMO) [7]. Retrobulbar neuritis accounts for 2/3 of ON [8]. In its atypical form, ON can present with a painless visual loss and be associated with previous history of neoplasia, which matches our patient's case (Figure 4). NMO doesn't seem specific for an autoimmune disease in particular. In the study led by Pittock in 2008, 50\% of the patients with NMO were seropositive for NMO-IgG (whose antigen is aquaporin-4) without coexisting Sjögren syndrom (SS) or systemic lupus erythematosus (SLE).

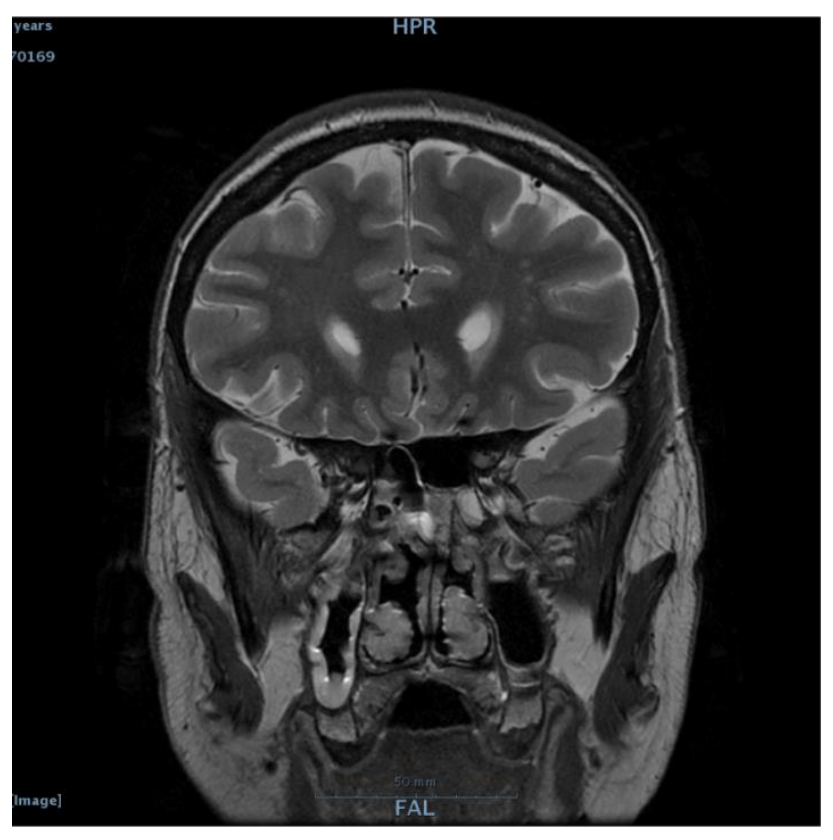

Figure 4: T2 coronal centered on the optic nerve: hyper signal of the left optic nerve in the intracanalicular section.

Moreover, non-organ-specific antibodies were frequently encountered in patients with NMO (25\%). In our case, the patient showed no signs of MS, SS or SLE. Biologically, there was no specificity of the autoantibodies but the NMO-IgG hasn't been sought.

It appears that CNS may be the target of cGvHD and thus, must be taken care accordingly. In 2014, quite a similar case was described in the Neuro-ophthalmology Journal, based on a 21-year-old woman who presented with a subacute, painless visual loss, six months after an allogeneic HSCT for Hodgkin disease. The ophthalmic examination and the MRI were both normal. An inflammatory cause was considered and the visual field partially improved after high-dose intravenous methylprednisolone [9]. Zheng et al. [10] also reported a case of ON (ptosis then mild sicca symptoms) and longitudinally extensive transverse myelitis, within an autoimmune context (anti-nuclear, anti-SSA, anti-SSB antibodies and NMO-IgG positive) successfully treated with tacrolimus after failure of high dose of oral prednisolone and intravenous cyclophosphamide.

Herein we report the case of a rare clinical form of cGvHD with central neurological damage focused on the optic nerve with positive and complete response to immunosuppressive tacrolimus therapy.

\section{References}


www.tridhascholars.org | April-2018

1. Filipovich AH, Weisdorf D, Arora M, et al. (2005) National Institutes of Health consensus development project on criteria for clinical trials in chronic graft-versus-host disease: I. Diagnosis and staging working group report. Biology of Blood and Marrow Transplant 1(12): 945-956.

2. Grauer O, Wolff D, Bertz H, et al. (2010) Neurological manifestations of chronic graft-versus-host disease after allogeneic haematopoietic stem cell transplantation: report from the Consensus Conference on Clinical Practice in chronic graft-versus-host disease. Brain 133(10): 2852-2865.

3. Socié G and Ritz J (2014) Current issues in chronic graft-versus-host disease. Blood Journal 124: 374-385.

4. S. Thépot and G. Socié (2010) Graft versus host disease: pathophysiology and first line therapy. Onco-hématologie 1.

5. Harvey CM, Gottipati R, Schwarz ST, et al. (2014) Acute disseminated encephalomyelitis following allo-SCT: central nervous system manifestation of GvHD. Bone Marrow Transplant. 49(6): 854-856.

6. Pittock SJ, Lennon VA, Seze JD, et al. (2008) Neuromyelitis optica and non-organ-specific autoimmunity. Archives of Neurology. 65(1): 78-83.

7. Menon V, Saxena R, Misra R, et al. (2011) Management of optic neuritis. Indian Journal of Ophthalmology 59(2): $117-122$.

8. Hoorbakht $\mathrm{H}$ and Bagherkashi $\mathrm{F}$ (2012) Optic neuritis, its differential diagnosis and management. Open Ophthalmology Journal 6: 65-72.

9. Moesen I and Kidd DP (2014) Bilateral Inflammatory Optic Neuropathy Related to Graft versus Host Disease Following Allogeneic Bone Marrow Transplantation for Hodgkin Disease. Neuro-Ophthalmology 38(4): 224-229.

10. Zheng X, Zhang X, Liu X, et al. (2014) Patient with neuromyelitis optica spectrum disorder combined with Sjögren's syndrome relapse free following tacrolimus treatment. Internal Medicine. 53(20): 2377-2380. 